\title{
Parabole aziendali e innovazione comunicativa La storia del cappellificio Borsalino
}

\section{di Cinzia Bianchi}

Università di Modena e Reggio Emilia, Dipartimento di Comunicazione ed Economia cinzia.bianchi@unimore.it

www.cinziabianchi.it

\begin{abstract}
In questo saggio viene presentato un caso studio riguardante la marca Borsalino, la cui evoluzione comunicativa si intreccia strettamente con la storia aziendale. Rimangono presupposti imprescindibili di questo breve lavoro sia la storia della pubblicità e soprattutto della cartellonistica della prima metà del Novecento, sia la metodologia semiotica che ci ha permesso da una parte di scegliere un corpus coerente ma limitato di immagini da confrontare e dall'altra di individuare elementi testuali costanti e variabili, adottando un punto di vista prevalentemente diacronico.

This paper presents a case study of the brand 'Borsalino', whose communication strategy has evolved closely intertwined with the history of the company. The theoretical foundations of the present work stand in the history of advertising, particularly in the first half of the twentieth century, and in a semiotic approach which has allowed us to choose a coherent corpus of images, to compare them and to identify textual constants and variables, within a diacronic perspective.
\end{abstract}

\section{Parole chiave}

Pubblicità, innovazione, immagine, diacronia, Borsalino 
Gli studiosi di pubblicità sanno benissimo che ci sono momenti della vita di un'azienda, non sempre legati a vicende apparentemente positive, che possono divenire veri e propri motori di innovazione comunicativa ${ }^{1}$. Mi è capitato recentemente di verificare questo principio generale analizzando la comunicazione pubblicitaria della Borsalino, nota fabbrica di cappelli di Alessandria.

Fondata nel 1857 da Giuseppe Borsalino con il fratello Lazzaro, l'azienda attraversa un momento particolarmente complicato quando uno degli eredi, con alcuni altri imprenditori italiani, fonda la Borsalino fu Lazzaro \& Co., sempre fabbrica di cappelli e con sede ancora ad Alessandria. Siamo nel 1906 e per una trentina di anni i famosi cappelli Borsalino saranno venduti da due aziende che condividono parte del nome e un'importante reputazione da difendere. È questa una storia relativamente breve della prestigiosa fabbrica di cappelli che dura fino a quando la "nuova" Borsalino non viene riassorbita nel 1938, dopo la crisi economica del 1929 e una lunga trattativa legale e commerciale, dalla casa madre. ${ }^{2}$ Di questi trent'anni risulta particolarmente interessante per uno studioso di linguaggi pubblicitari il fatto che le due Borsalino si fronteggino scegliendo anche l'ambito della comunicazione.

È infatti un manifesto pubblicitario di Cesare Simonetti a sancire nel 1906 la divisione dell'azienda anche dal punto di vista comunicativo: due cani che si contendono un cappello sono al centro della scena, contornati da diverse scritte tra le quali emerge proprio il nome della nuova fabbrica di cappelli e la sua dislocazione (cfr. fig. 1). Se questo tipo di informazione sembra essere lo scopo principale del cartellone, non si deve tuttavia sottovalutare la necessità di ribadire alcune caratteristiche proprie del prodotto, come la resistenza evidenziata sia dal punto di vista visivo attraverso il gioco dei cani, sia dal punto di vista verbale. Più volte ripreso negli anni successivi, tale manifesto è sicuramente emblematico della nuova fase che le due Borsalino dovranno affrontare.

1 Il termine "innovazione", così come "innovatività", spessissimo utilizzato nel mondo pubblicitario, rimane difficilmente definibile. Spesso lo si preferisce sostituire con "invenzione di nuove tecniche comunicative per pubblicizzare un prodotto" oppure con "creatività". Rimane in ogni caso un alone di indefinito e di misterioso su cui un'analisi semantica approfondita potrebbe fornire brillanti riflessioni. Per quanto riguarda lo scopo di questo saggio, direi di accontentarci di una definizione meno tecnica, utilizzando "innovazione" e "innovatività" soprattutto nella prospettiva di evoluzione del linguaggio pubblicitario, quindi dal punto di vista prevalentemente di storia della pubblicità.

${ }_{2}^{2}$ Tutto ha infatti inizio con il "Mastro Cappellaio" Giuseppe Borsalino che nel 1857 apre con il fratello Lazzaro la prima follatura ad Alessandria. Giuseppe sembra essere il vero motore dell'azienda che cresce rapidamente grazie alla qualità del prodotto e alla costante ricerca di nuovi mercati, in Europa innanzitutto, ma anche in Sud America, negli Stati Uniti, in Australia e Nuova Zelanda. Nel 1900, alla morte del padre, Teresio Borsalino eredita la fabbrica ma suo cugino Giovanni Battista, estromesso dal nuovo assetto societario dell'azienda di famiglia, decide di aprire ad Alessandria un proprio cappellificio, sotto il nome "Borsalino fu Lazzaro \& Co". Inizia così uno scontro commerciale e comunicativo tra i due cappellifici che termina solo nel 1938, quando la "nuova” Borsalino verrà riassorbita dalla casa madre. 


\section{Ocular flux_saggi}

Parabole aziendali e innovazione comunicativa | Cinzia Bianchi

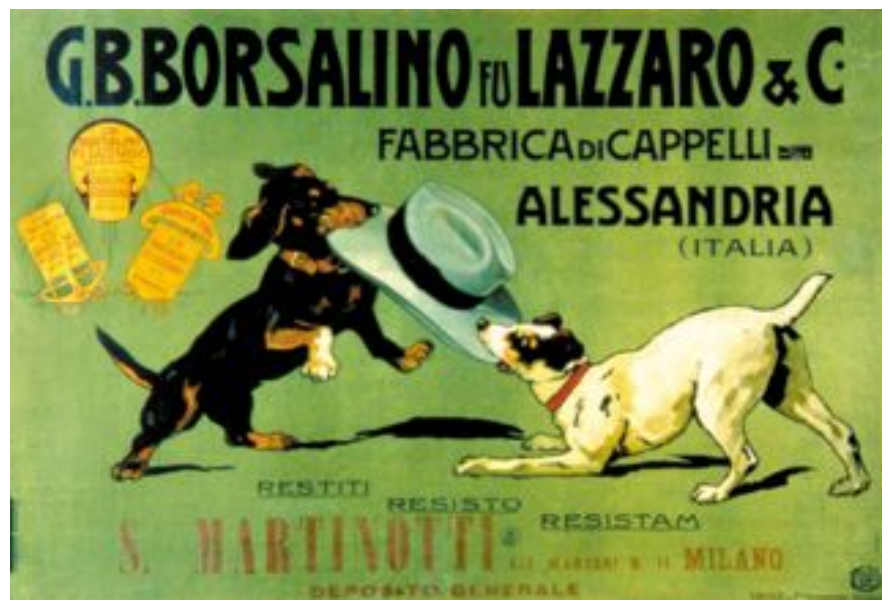

Fig. 1. Manifesto di Cesare Simonetti per Borsalino fu Lazzaro \& C. (1906)

È interessante confrontare tale manifesto con una versione di qualche anno dopo, dove si è lavorato a creare una maggiore chiarezza di lettura (cfr. fig. 2). Rimangono identici immagine, nome dell'azienda in particolare evidenza e il messaggio, ma cambia il colore dello sfondo per creare maggiore contrasto e scompaiono gran parte delle altre scritte a vantaggio di una maggior nitidezza d'insieme.

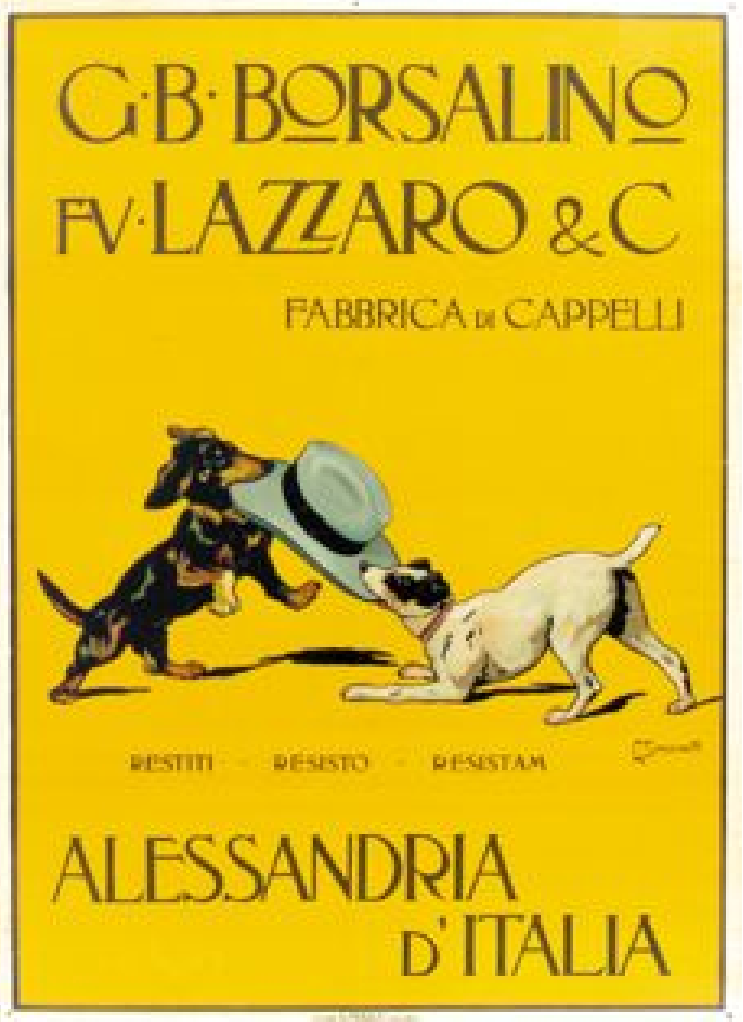

Fig. 2. Manifesto di Cesare Simonetti per Borsalino fu Lazzaro \& C. (1913) 


\section{Ocula $a^{\text {Flux__aggi }}$}

Parabole aziendali e innovazione comunicativa | Cinzia Bianchi

La Borsalino fu Lazzaro sembra captare un'opportunità che l'ambito dell'arte offre in quegli anni alla comunicazione aziendale. Infatti, come ci racconta Elio Grazioli nel suo libro Arte e pubblicità, "arte moderna e pubblicità si può dire che nascano contemporaneamente". Si ha cioè l'evoluzione in parallelo della figura dell'artista e del pubblico, causata da una parte dai "grandi cambiamenti sociali, tecnologici e distributivi che hanno comportato un'irreversibile trasformazione dei caratteri e dei principi stessi dell'arte"; e dall'altra da "rivoluzione industriale, riproducibilità tecnica, moltiplicazione dei prodotti e loro trasformazione in merce, aumento del consumo e formazione delle masse, diffusione dell'informazione e costruzione della rete di trasporti e distribuzione" (Grazioli, 2001, 5). ${ }^{3}$

Quello descritto da Grazioli è un processo che ha attraversato la nostra storia dell'ultimo secolo e mezzo e nel quale è possibile poi individuare alcuni momenti particolarmente fecondi, come l'epoca delle réclame, dei manifesti pubblicitari sparsi nelle città, della cartellonistica di inizio Novecento.

Per quanto riguarda la Borsalino fu Lazzaro, un passo decisivo in questa direzione si ha nel 1910 quando viene indetta una gara pubblica per pubblicizzare un nuovo prodotto, il cappello Zenit. Vince Marcello Dudovich ${ }^{4}$, un illustratore molto conosciuto nella prima parte del Novecento che propone un manifesto in cui il cappello è lasciato sulla sedia (cfr. fig. 3).

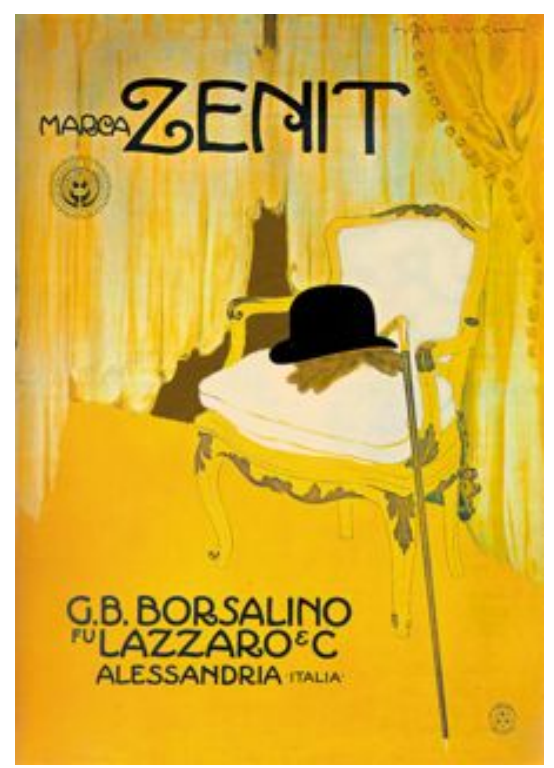

Fig. 3. Manifesto di Marcello Dudovich per Borsalino fu Lazzaro \& C. (1911).

\footnotetext{
3 Cfr. E. Grazioli, Arte e pubblicità, Bruno Mondadori, 2001.

4 Marcello Dudovich (1878-1962), insieme a Leonetto Cappiello, Adolf Hohenstein ed altri, è stato uno dei padri del moderno cartellonismo pubblicitario italiano. Fu un illustratore molto apprezzato per mezzo secolo, avendo ricevuto molti riconoscimenti internazionali, come una medaglia d'oro all'Esposizione Universale di Parigi del 1900. Oltre che per le due Borsalino, lavorò per Grandi Magazzini Mele (dal 19061911) e per la Rinascente (1921-1956) producendo un centinaio di manifesti nei quali rivisitò stili e mode a lui contemporanei, dal liberty al decò, contribuendo alla formazione del moderno linguaggio pubblicitario.
} 
In questo manifesto c'è un evidente contrasto cromatico tra l'oggetto pubblicizzato e il contesto: le tonalità calde del giallo caratterizzano i panneggi delle pareti e il pavimento, la poltroncina ambrata, i guanti e il bastone di un marrone chiaro, mentre il cappello è nero, così come lo sono le indicazioni della fabbrica produttrice e del particolare modello di cappello5. Completamente assente la figura umana, si tratta del primo esempio nella storia della pubblicità di un manifesto in cui il prodotto appare in primo piano ${ }^{6}$.

Sempre della Borsalino fu Lazzaro è un ulteriore prodotto comunicativo del 1912, un filmato strettamente correlato contenutisticamente a questa pubblicità. Si tratta di uno dei primi esempi di "cinematografia industriale" dove viene spiegata un'intera filosofia produttiva, mettendo in stretta correlazione immaginazione e creazione del cappello, la produzione del prodotto e la sua pubblicizzazione. Il filmato è suddiviso in tre macrosequenze, narrativamente molto diverse.

Nella prima si racconta di un naufrago su un'isola che per nutrirsi uccide un coniglio, lo cuoce e mentre lo sta mangiando scopre per caso una delle caratteristiche della sua pelle, l'estrema morbidezza e resistenza che si ottiene se entra in contatto con l'acqua. Questa parte del filmato si chiude con il primo piano del nostro naufrago che guarda in macchina intensamente con un mezzo sorriso, tanto da suggerirci che un'idea gli è sovvenuta.

Nella seconda macrosequenza ricompare lo stesso personaggio in un contesto completamente diverso: entrando nell'anticamera di una casa signorile, abbandona il suo bastone, i guanti e il cappello Borsalino su una poltroncina. Scomparso il personaggio dietro i tendaggi, l'immagine ripresa si trasforma nel manifesto di Dudovich presentato due anni prima (cfr. fig. 3).

La terza macrosequenza, quella più lunga, è il vero e proprio documentario industriale in quanto si mostrano tutte le singole fasi di produzione e lavorazione di un cappello Borsalino, dal trattamento del pelo per farne tessuto, la modellatura dei cappelli fino alle rifiniture e successiva spedizione.

L'intero filmato esplicita in modo lampante una filosofia aziendale del tutto innovativa: per una buona riuscita del prodotto è necessaria l'immaginazione e la creazione, a cui deve seguire una perfetta realizzazione, senza poter però trascurare la fase finale, cioè la pubblicizzazione del prodotto.

$\mathrm{Al}$ di là del pregio storico di questo esordio cinematografico della Borsalino fu Lazzaro, ci preme sottolineare come l'intero audiovisivo si possa anche interpretare come un tentativo di creare una storia narrata intorno a un'immagine, quella ideata da Dudovich, di per sé piuttosto innovativa e quindi probabilmente di difficile fruizione da parte del pubblico che l'azienda vuole il più vasto possibile. Ci sembra di poter infatti individuare i tratti caratteri-

\footnotetext{
5 Sebbene non sia lo scopo di questo saggio presentare analisi semiotiche delle singole immagini, sottolineiamo come potremmo procedere a un approfondimento del testo visivo che vada a individuare, oltre al livello "figurativo", anche quello cosiddetto "plastico", con le categorie cromatiche, eidetiche e topologiche. Per un ulteriore approfondimento semiotico cfr. Bianchi-Ragonese, L'annuncio pubblicitario, Carocci, 2013.

6 Per approfondire cfr. A. Abruzzese, F. Colombo (a cura di), Dizionario di pubblicità, Bologna, Zanichelli, $1^{\circ}$ ed. 1994 e il recente: V. Codeluppi, Storia della pubblicità italiana, Carocci, 2013.
} 


\section{Ocula $a^{\text {flux__Saggi }}$}

Parabole aziendali e innovazione comunicativa | Cinzia Bianchi

stici dell'eterno scontro/incontro tra la creatività del pubblicitario, che è un artista visivo, e le esigenze comunicative e divulgative delle aziende; in questo caso il filmato aiuta il reciproco avvicinamento tra ambiti produttivi che devono convivere e compenetrarsi in modo efficace.

E come reagisce l'altra Borsalino a tutto questo?

La Borsalino Antica Casa cerca da una parte di impedire alla rivale l'uso del nome dell'azienda, ormai sinonimo di cappello nel mondo; dall'altra però non può che accettare la sfida comunicativa e ingaggia gli stessi illustratori, primo tra tutti Dudovich. Durante gli anni Venti e Trenta, quest'ultimo firma diversi manifesti di entrambe le Borsalino, portando in primo piano il suo talento e, molti sostengono, anche una buona dose di opportunismo (cfr. figg. 4 e 5,6 e 7 ).
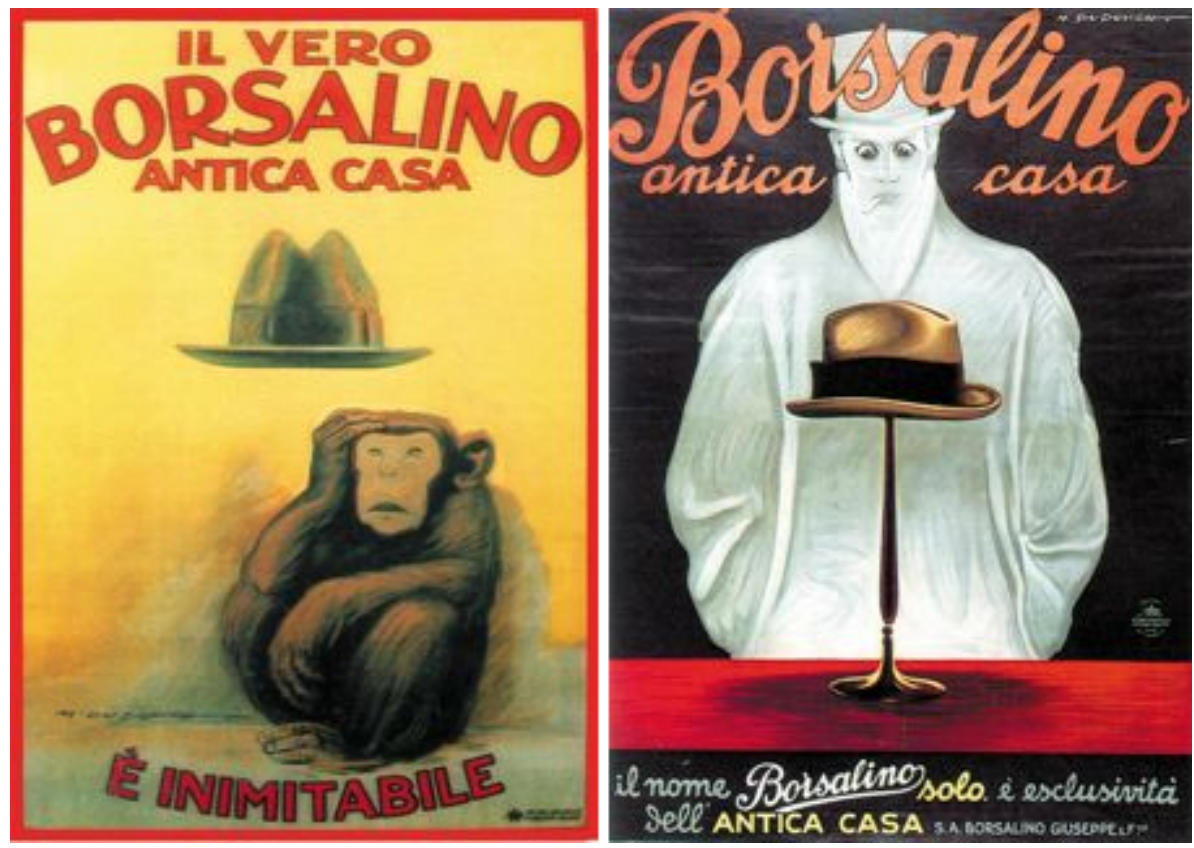

Figg. 4 e 5. Manifesti di Marcello Dudovich per Borsalino Antica Casa (1921 e 1924/28). 


\section{Oculartux_saggi}

Parabole aziendali e innovazione comunicativa | Cinzia Bianchi
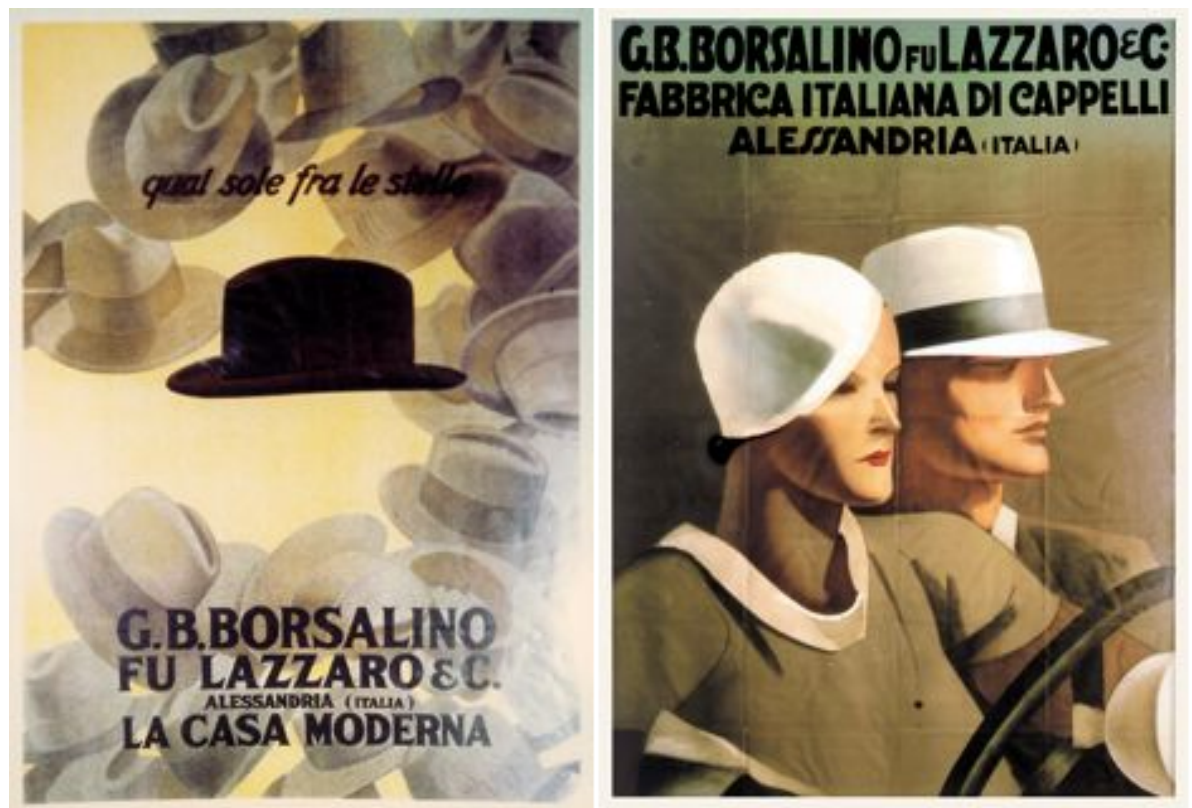

Figg. 6 e 7. Manifesti di Marcello Dudovich per Borsalino fu Lazzaro \& C. (1930)

Se confrontiamo i manifesti che Dudovich ha ideato per le due Borsalino (cfr. figg. 4-5 e 6-7) non emergono particolari differenze dal punto di vista di cura formale e ideazione. Si potrebbe ritenere che la più innovativa dal punto di vista della storia della pubblicità sia la $\mathrm{n}^{\circ} 6$, dove spicca un cappello nero in mezzo a una corona di altri cappelli di color beige. Lo slogan: "quel sole fra le stelle" evidenzia il ruolo preminente del cappello Borsalino, che emerge nella sua lucentezza tra gli altri cappelli così come, per similitudine, il sole tra le stelle. Come nel manifesto del 1911 (cfr. fig. 3), il prodotto è in primo piano, così come nei manifesti della Borsalino Antica Casa (cfr. figg. 4 e 5), dove però non si rinuncia alla presenza di qualche altro soggetto secondario, come la scimmietta e il fantasma.

Ma ciò che maggiormente colpisce di questi due ultimi manifesti è la parte verbale. Gli slogan: "è inimitabile" e "il nome Borsalino solo è esclusività dell'Antica Casa" indicano chiaramente la volontà del committente, intenzionato a sfruttare la comunicazione pubblicitaria per contrastare i diretti concorrenti, ribadendo valori di primogenia e competenza "antica". Tale valorizzazione della tradizione viene contrastata dalla innovatività esplicitamente dichiarata dalla Borsalino fu Lazzaro con lo slogan: "la casa moderna" (cfr. fig. 6)7. Da un confronto tra le due comunicazioni d'azienda si ha infatti

7 Il confronto tra queste quattro immagini, scelte appositamente per mostrare le diverse strategie comunicative delle due Borsalino, potrebbero essere approfondite dal punto di vista semiotico, approntando una complessa analisi del livello dell'espressione e del contenuto. Come abbiamo già ribadito, non è questo lo scopo del saggio, ma piuttosto vogliamo fornire spunti metodologici per ulteriori approfondimenti sia dal punto di vista sincronico che diacronico. Per approfondire l'analisi sincronica e diacronica degli annunci-stampa, cfr. Bianchi-Ragonese, L'annuncio pubblicitario, Carocci. 2013, capp. 4 e 5. 


\section{Ocular flux_saggi}

Parabole aziendali e innovazione comunicativa | Cinzia Bianchi

l'impressione di una maggiore innovazione e creatività di quest'ultima che ingaggia anche altri illustratori importanti come Giuseppe Minonzio e Gino Boccasile $^{8}$.

I manifesti che presentiamo (figg. 8 e 9) sono due esempi della ricerca di nuove forme e contenuti da associare al prodotto pubblicizzato. Il cappello è sempre in primo piano e, giocando con la conchiglia o con una nota musicale, viene metaforicamente associato alla preziosità oppure all'eleganza del portamento.
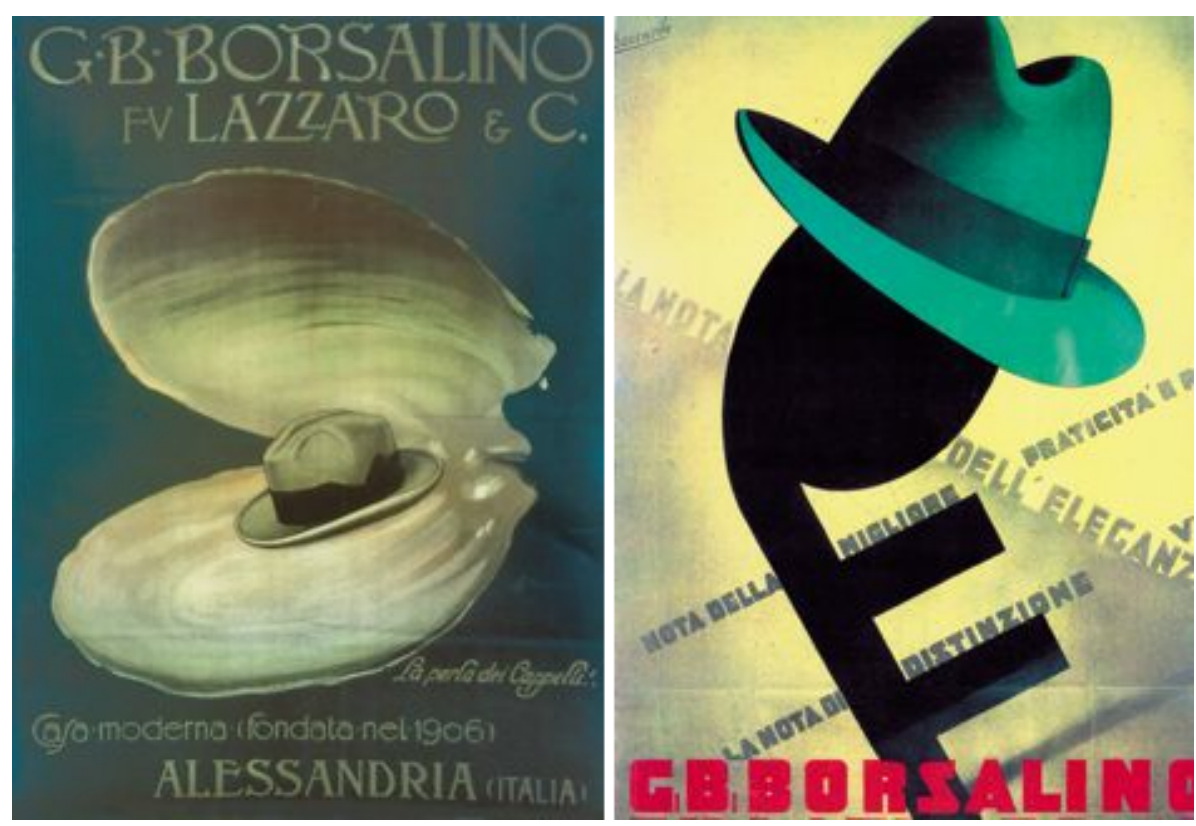

Figg. 8 e 9. Manifesto di Giuseppe Minonzio (1920) e Gino Boccasile (1933) per Borsalino fu Lazzaro \& C.

È questo in generale un periodo in cui le imprese riservano un'attenzione pionieristica alla comunicazione, e si comincia a consumare l'eterna dialettica tra "apporto creativo dell'artista, sua audacia avanguardista e tentativo di sperimentazione dell'industria o addomesticamento delle scoperte". Come bene sintetizza Grazioli, "da un lato illuminati impresari si accostano alle ultime forme dell'arte e anzi incitano gli artisti che amano osare e inventare, dall'altro le esigenze di raggiungere un pubblico vasto ed eterogeneo, e di convincerlo ad acquistare piuttosto che a contemplare o capire, frenano o accomodano di fatto le intenzioni” (Grazioli, 2001, p. 33).

La comunicazione delle due Borsalino si inserisce a pieno titolo in questo interessante capitolo della storia della pubblicità; l'attenzione all'innovazione

${ }^{8}$ Gino Boccasile (1901-1952) fu un cartellonista e pittore che, dopo aver lavorato in Francia e Argentina, lavorò in Italia a partire dagli anni Trenta. Realizzò un gran numero di manifesti anche per molte altre aziende italiane (Yomo, La Rinascente, Locatelli, Moretti, Paglieri e Pirelli) e, unico tra gli illustratori italiani, creò anche manifesti per la propaganda politica e bellica. 


\section{Ocular flux_saggi}

Parabole aziendali e innovazione comunicativa | Cinzia Bianchi

e alla creatività comunicativa è stata favorita inizialmente da questa divisione dell'azienda e dalla sfida tra le due Borsalino, ma è poi continuata successivamente per molti anni dopo la riunificazione. Pensiamo per esempio alle campagne di fine anni Quaranta in cui Max Huber esprime il suo innovativo stile grafico oppure alla famosissima immagine in stile divisionista di Armando Testa del 1954.
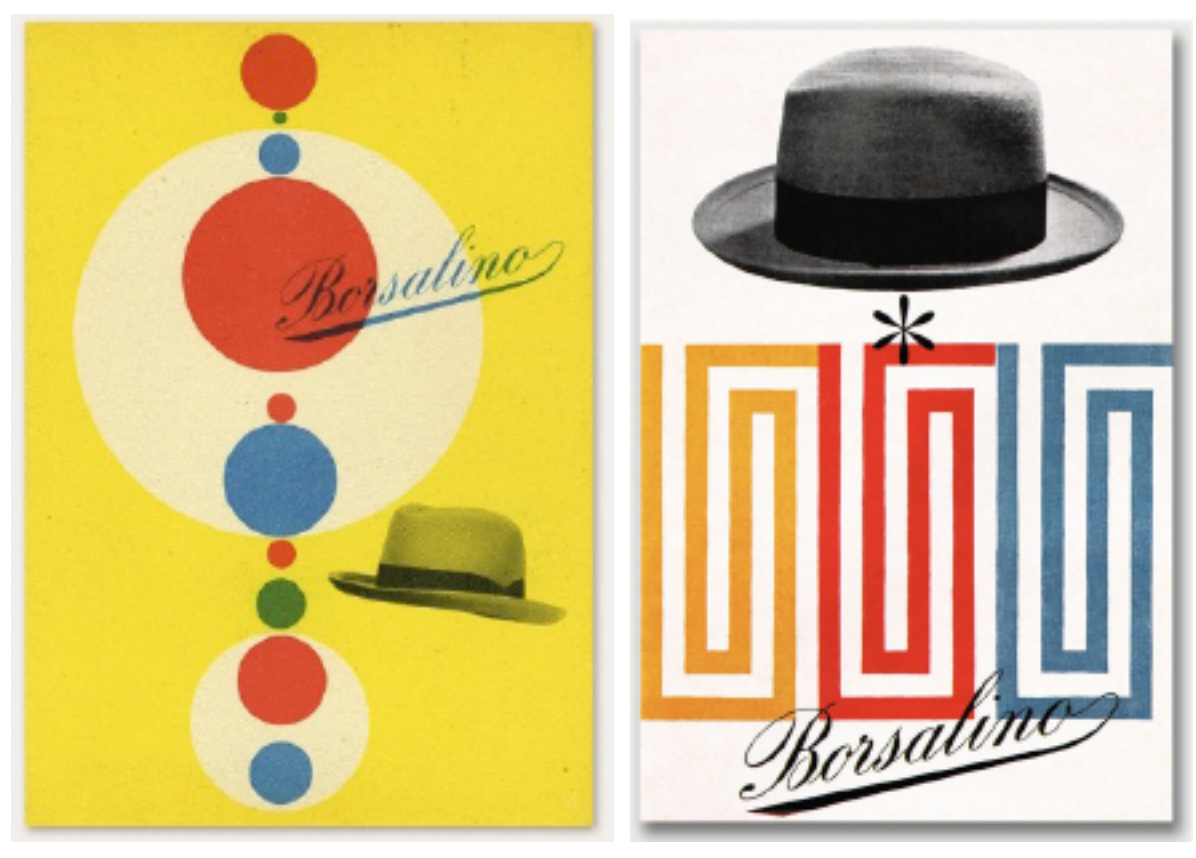

Figg. 10 e 11. Manifesti di Max Huber per Borsalino (1948-49).

Max Huber ${ }^{9}$ è notoriamente l'artista grafico che ha introdotto la tecnica del fotomontaggio nell'immagine pubblicitaria. Le figure 10 e 11 sono solo due esempi della sua vasta produzione di manifesti e bozzetti elaborati per Borsalino. Lavorando su pochi elementi essenziali, Huber compone i suoi manifesti pubblicitari evitando quasi sempre lo slogan; è in bella evidenza il marchio logogrammatico in bella grafia rotondeggiante, una fotografia del cappello, con un suo punto di illuminazione e la sua ombra, e infine un motivo grafico predominante. Nella figura 10 si tratta di cerchi di diversi colori e grandezze disposti sull'asse verticale, mentre nella 11 di una greca stilizzata vagamente classicheggiante. Da notare come in quest'ultima ci sia un asterisco sotto al cappello, quasi a indicare il soggetto assente dall'immagine che però è colui a cui è rivolto il messaggio complessivo: è, per così dire, una presenza inusuale nell'enunciato del consumatore/destinatario, ciò che in se-

9 Max Huber (1919-1992) è un designer di grande talento e personaggio di spicco della grafica pubblicitaria novecentesca. Fa parte del gruppo di grafici europei che hanno lavorato negli anni Quaranta e Cinquanta intorno allo Studio Boggeri, sperimentando nuove tecniche comunicative. Tra l'altro, ha realizzato a partire dal $1947 \mathrm{e}$ per diversi anni la grafica delle pubblicazioni Einaudi, e ha creato nel 1950 il marchio della Rinascente. 
miotica si intende con marca dell'enunciazione..$^{10}$ Lo stile di Huber ha una sua riconoscibilità identitaria; da parte sua, la Borsalino non fa altro che assecondare la creatività del grafico che liberamente gioca anche a rimandi stilistici e citazioni ben evidenti.

Pochi anni dopo, Borsalino commissiona a un altro pubblicitario importante, Armando Testa ${ }^{11}$, una nuova campagna pubblicitaria, la cui immagine fondamentale è quella di un uomo puntinato in bianco e nero con il cappello sospeso (cfr. fig. 12). Anche qui sono evidenti i rimandi stilistici, in particolare al divisionismo di Seurat, e il contrasto, cromatico ed eidetico, che si crea tra questa immagine dell'uomo e quella, molto realistica, del cappello sospeso. Molti anni dopo, l'autore riconosce che questa è un'immagine "senza tempo" di cui si sente molto orgoglioso: è riuscito a inventare un'immagine che potrebbe essere stata ideata in un tempo precedente o successivo e ciò gli creava ancora, negli anni Ottanta, una certa emozione.

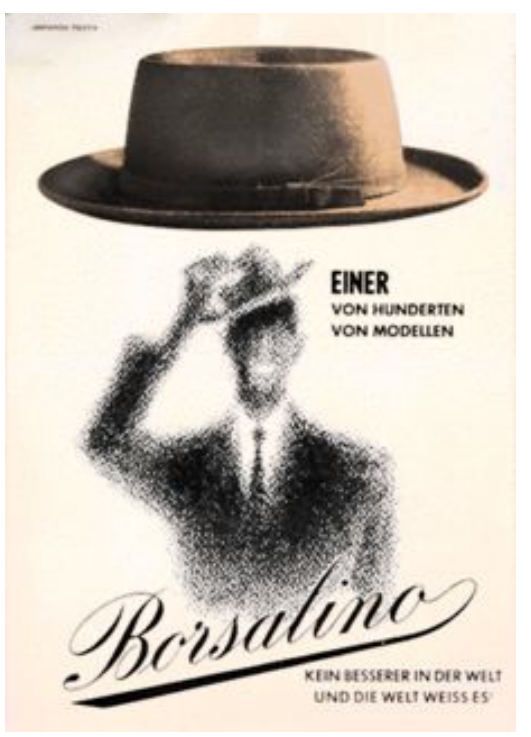

Fig. 12. Manifesto di Armando Testa per Borsalino (1954).

${ }^{10}$ Una parte importante degli studi semiotici sull'Enunciazione riguardano il testo visivo. Così come esistono in un testo scritto tracce di un soggetto enunciatore e di un enunciatario inscritto, allora analoghe tracce devono esistere anche in un testo visivo. Gli studi sull'enunciazione visiva si sono concentrati inizialmente sul testo pittorico, ma sono presto arrivati a comprendere anche testi audiovisivi e pubblicitari. L'enunciatore può essere presente nel testo attraverso delle strategie simulacrali, come lo sguardo verso l'enunciatario oppure, nel caso della pubblicità, la presenza stessa del logo, vera e propria marca dell'enunciazione (come nel nostro caso la scritta "Borsalino"). Del resto ci possono essere strategie di inclusione nel testo dello stesso enunciatario, come in questo caso questo asterisco che richiama una parte del corpo del potenziale consumatore. Per approfondire il concetto di Enunciazione cfr. G. Manetti, L'enunciazione, Mondadori, 2008 (prima ed. Protagon 1998)

11 Armando Testa (1917-1992) è un pittore e cartellonista con uno stile grafico decisamente innovativo e spesso ironico. I suoi primi manifesti pubblicitari risalgono al 1945. Alla fine degli anni Quaranta apre uno studio a Milano, poi divenuto la più importante agenzia pubblicitaria italiana. Sono sue le campagne della Lavazza con i personaggi di El Caballero e Carmensita, così come l’ippopotamo Pippo della Lines, "la pancia non c'è più" per l'olio Sasso, la bionda della birra Peroni, la campagna Punt e Mes, e così via. 


\section{Oculartux_saggi}

Parabole aziendali e innovazione comunicativa | Cinzia Bianchi

Un capitolo a parte della comunicazione della Borsalino riguarda lo stretto collegamento con l'industria cinematografica hollywoodiana che, a partire dagli anni Venti, costruisce un indubbio canale di pubblicizzazione del prodotto e della marca. Nel nuovo star system non c'è nessun gangster senza il suo cappello e molti di loro, come Lucky Luciano, Al Capone o John Dillinger indossano un Borsalino. Così come poliziotti ed investigatori privati, che non possono fare a meno del loro fedora, il cappello a tesa larga, la cui ombra crea personaggi misteriosi. Diffusosi rapidamente in tutti i generi cinematografici, il cappello Borsalino è a lungo presente nell'immaginario collettivo, è il cappello per antonomasia, possiamo riferirci ad esso senza che l'azienda abbia la necessità di intraprendere nessuno sforzo per incentivare altra creatività.
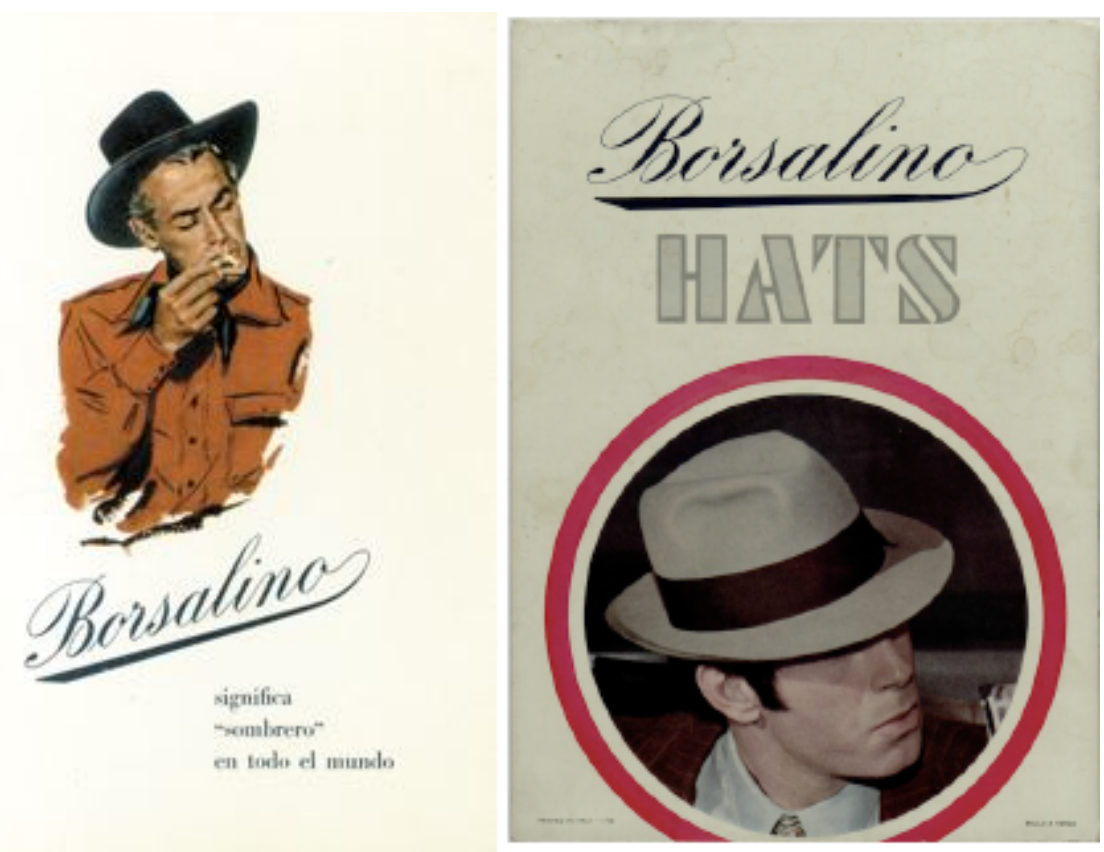

Figg. 13 e 14. Due annunci-stampa Borsalino degli anni Cinquanta e Sessanta.

Questi anni costituiscono il momento di maggiore notorietà del cappello Borsalino, che dovrà però far fronte presto a un cambiamento di abitudini vestimentarie che non prevedono un utilizzo costante del cappello. A questo dato commerciale si aggiunge però, a nostro avviso, anche una mancata sensibilità ai nuovi linguaggi pubblicitari. Sono questi gli anni di Carosello e seguiranno presto quelli dell'audiovisivo pubblicitario vero e proprio con i suoi spot; un linguaggio emergente allora che molto peso ha avuto ed ha ancora nella cultura di massa.

La comunicazione Borsalino rimane a lungo legata alle bellissime immagini della prima parte del Novecento, riproposte come un unico comunicativo, senza far particolarmente attenzione al fatto che la loro innovazione è legata a una dolorosa storia aziendale. Sono queste il suo maggior vanto creativo ma allo stesso tempo la sua maggiore debolezza per il futuro: ap- 
prezzare e valorizzare il passato non sempre permette di captare le novità del presente e del futuro.

Questa breve storia dell'azienda Borsalino e della sua comunicazione pubblicitaria ci permette di compiere almeno due tipi di riflessioni.

La prima e più generale riguarda la storia della pubblicità italiana di cui Borsalino è stata protagonista almeno fino alla metà del Novecento, facendo parte di quella decina di marchi aziendali che si sono caratterizzati per essere veri e propri motori finanziari della sperimentazione di nuovi linguaggi pubblicitari. I passaggi fondamentali di questo percorso della Borsalino possono essere letti in parallelo con quelli di altri marchi, che hanno utilizzato spesso anche gli stessi artisti grafici e pubblicitari. Riteniamo doveroso evidenziare come nella nostra ricostruzione sia stata particolarmente fruttuosa un'attenzione verso i testi visivi compiuta attraverso un approccio semiotico che ci ha permesso di individuare, ricostruire ed evidenziare quei momenti di cambiamento, di vera e propria trasformazione comunicativa; sebbene non siano state presentate approfondite analisi semiotiche, la scelta del corpus e le domande poste ai singoli testi mostrano come sia possibile adottare un punto di vista diacronico, e non solo prettamente sincronico, anche con il solo supporto degli strumenti propri dell'analisi narratologica e visiva (cfr. Bianchi-Ragonese 2013).

La seconda riflessione riguarda in specifico l'identità della marca Borsalino che ha teso progressivamente a dividere la storia aziendale, fatta anche, come abbiamo visto, di contrasti ereditari profondi, dalla sua storia pubblicitaria, vissuta e riproposta nei decenni successivi al 1938 come un insieme omogeneo di prodotti comunicativi innovativi e rilevanti nel panorama italiano e internazionale. Questo aspetto è molto interessante perché in realtà è stata proprio la suddivisione dell'azienda a rendere necessaria la costruzione di un'identità comunicativa forte delle due Borsalino, le quali hanno lavorato a differenziare la comunicazione di marca attraverso un processo di continuità (legato soprattutto all'identità parziale del nome e alla buona fattura del prodotto) e di discontinuità valoriale (una Borsalino si presentava come il "nuovo" mentre l'altra come l'“antica casa"; l'una ribadiva valori di "modernità" e l'altra di "inimitabilità"; e così via). L'opposizione semantica profonda tra /innovazione/ e /tradizione/ ha caratterizzato questa fase della vita delle Borsalino, e proprio questo contrasto così netto è stato il vero motore di innovazione comunicativa. La Borsalino di nuovo unificata ha ritenuto necessario far tesoro di tutto questo ricco patrimonio: dal punto di vista valoriale ha consolidato l'attenzione all'unicità del prodotto e alla sua buona fattura, mentre dal punto di vista comunicativo ha fatto proprio lo spirito innovativo che caratterizzava soprattutto la Borsalino fu Lazzaro. Si tratta di un mix di valori e tecniche pubblicitarie che hanno costituito a lungo l'identità della marca Borsalino, e vengono considerati tuttora, a torto o a ragione, così forti nell'immaginario collettivo da poter resistere nel tempo ai cambiamenti correnti; quanto a lungo tutto ciò può ancora avere una ragionevole consistenza, lo potremmo capire solo nel futuro. 


\section{Oculartux_saggi}

Parabole aziendali e innovazione comunicativa | Cinzia Bianchi

\section{Bibliografia}

Abruzzese Alberto; Colombo Fausto (a cura di)

1994 Dizionario della pubblicità, Bologna, Zanichelli.

Bianchi Cinzia; Ragonese Ruggero

2013 L'annuncio pubblicitario, Roma, Carocci.

Codeluppi Vanni

2013 Storia della pubblicità italiana, Roma, Carocci.

Grazioli Elio

2001 Arte e pubblicità, Milano, Bruno Mondadori.

Manetti, Giovanni

2008 L'enunciazione, Milano, Mondadori (1 ${ }^{\circ}$ ed. Protagon, 1998). 